9. Papaemmanuil E, Gerstung M, Bullinger L, et al. Genomic classification and prognosis in acute myeloid leukemia. N Engl J Med. 2016;374 (23):2209-2221.

10. Saultz JN, Garzon R. Acute myeloid leukemia: a concise review. J Clin Med 2016:5(3)

11. Dombret H, Gardin C. An update of current treatments for adult acute myeloid leukemia. Blood 2016;127(1):53-61.

12. Nowak D, Stewart D, Koeffler HP. Differentiation therapy of leukemia: 3 decades of development. Blood 2009;113(16):3655-3665.

13. Sanz MA, Grimwade D, Tallman MS, et al. Management of acute promyelocytic leukemia: recommendations from an expert panel on behalf of the European LeukemiaNet. Blood 2009;113(3):1875-1891

14. DiNardo CD, Stein EM, de Botton S, et al. Durable remissions with ivosidenib in IDH1-mutated relapsed or refractory AML. N Engl J Med. 2018;378(25):2386-2398
15. Sykes DB, Kfoury YS, Mercier FE, et al. Inhibition of dihydroorotate dehydrogenase overcomes differentiation blockade in acute myeloid leukemia. Cell 2016;167(1):171-186.

16. Dang CV. MYC on the path to cancer. Cell. 2012;149(1):22-35.

17. Itkonen HM, Minner S, Guldvik IJ, et al. O-GlcNAc transferase integrates metabolic pathways to regulate the stability of c-MYC in human prostate cancer cells. Cancer Res. 2013;73(16):5277-5287.

18. Jozwiak P, Forma E, Brys M, Krzeslak A. O-GlcNAcylation and metabolic reprograming in cancer. Front Endocrinol (Lausanne) 2014;5:145.

19. Ladds $M$, van Leeuwen IMM, Drummond CJ, et al. A DHODH inhibitor increases p53 synthesis and enhances tumor cell killing by p53 degradation blockage. Nat Commun. 2018;9(1):1107.

20. Cao L, Branstrom A, Baird J, et al. PTC299 is a novel DHODH inhibitor that modulates VEGFA mRNA translation and inhibits proliferation of a broad range of leukemia cells. Blood. 2017;130(Suppl 1):1371.

\title{
The complexity of stem cell transplants: can we improve our understanding?
}

\section{Andrea Bacigalupo ${ }^{1}$ and Francesca Bonifazi ${ }^{2}$}

'Fondazione Policlinico Universitario Gemelli IRCCS, Roma and 'Istituto di Ematologia "Seràgnoli", Azienda Ospedaliera Universitaria Sant'Orsola, Bologna, Italy

E-mail:apbacigalupo@yahoo.com

doi:10.3324/haematol.2018.198010

C ox regression analysis can be considered a robust, easy and universal way to calculate the role of variables on outcome endpoints, such as survival, disease-free survival, and so on. The Cox model is a semiparametric approach based on the strong assumption that the effects of different variables on survival (or on the particular endpoint) are constant over time and are additive in a particular scale.

The setting of allogeneic stem cell transplantation is, however, complicated by two additional levels that limit the application of Cox analysis and call for new, more complex, statistical methods: the first is that some variables in allogeneic stem cell transplantation are not timefixed covariates (such as age, gender, or type of donor) but develop after a certain interval of time from transplantation, and need to be accounted for as time-dependent. In other words, with a starting population of patients, some will develop an event (e.g., cytomegalovirus infection) and some will not: a comparison of patients with and without cytomegalovirus infection will need to consider the infection as a time-dependent variable.

A further level of complexity is provided by competing events: a competing event is one that precludes the event of interest from occurring, or significantly changes its probability. Death before cytomegalovirus infection, is a clear example of a competing event for cytomegalovirus infection. Relapse and non-relapse mortality is another clear example of competing events.

So, there are time-fixed covariates, time-dependent events, and competing events.

In a study published in this issue of Haematologica, Fuerst and colleagues have added a fourth level of complexity: they hypothesized that the effect of different covariates may be different at different intervals from transplantation, and this is exactly what they found.

One example is the stem cell source: bone marrow and peripheral blood as sources of stem cells have been compared in numerous prospective and retrospective studies, including meta-analyses, to define which is better, and results have often been conflicting. Again the complexity of transplantation does not make comparisons easy: in the first randomized study ${ }^{2}$ of patients with low-risk disease, receiving a myeloablative regimen and HLA identical sibling grafts, the hazard risk (HR) of death was 1.20 for recipients of peripheral blood compared to bone marrow $(P=0.2)$. In a more recent prospective study ${ }^{3}$ with unrelated donor grafts, using both myeloablative and reduced intensity conditioning regimens for patients with low, intermediate and high-risk disease, the risk of death was 1.20 for bone marrow versus peripheral blood $(P=0.2)$.

Fuerst and colleagues offer a new way of looking into this particular issue: they found that peripheral blood has a significant protective effect on non-relapse mortality early after transplantation, and a significant detrimental effect later on. ${ }^{1}$ The time point for a change of effect on non-relapse mortality was set at 8 months: this means that patients receiving peripheral blood grafts had a lower non-relapse mortality within 8 months (HR: 0.75) and a higher non-relapse mortality beyond 8 months after transplantation (HR:1.38), which were both highly significant effects (Figure 1). There was no protective effect of peripheral blood on relapse, which is the competing event (Figure 1). The authors also looked at a second model of competing events (transplant-related mortality and non-transplant related, or death due to other causes, including relapses), disproving common beliefs; they found no protective effect of peripheral blood as compared to bone marrow grafts on deaths due to other causes, which raises the question of whether peripheral blood should remain the preferred stem cell source in allogeneic stem cell transplants. Indeed an increased risk of chronic graft-versus-host disease seems not to be compensated by 


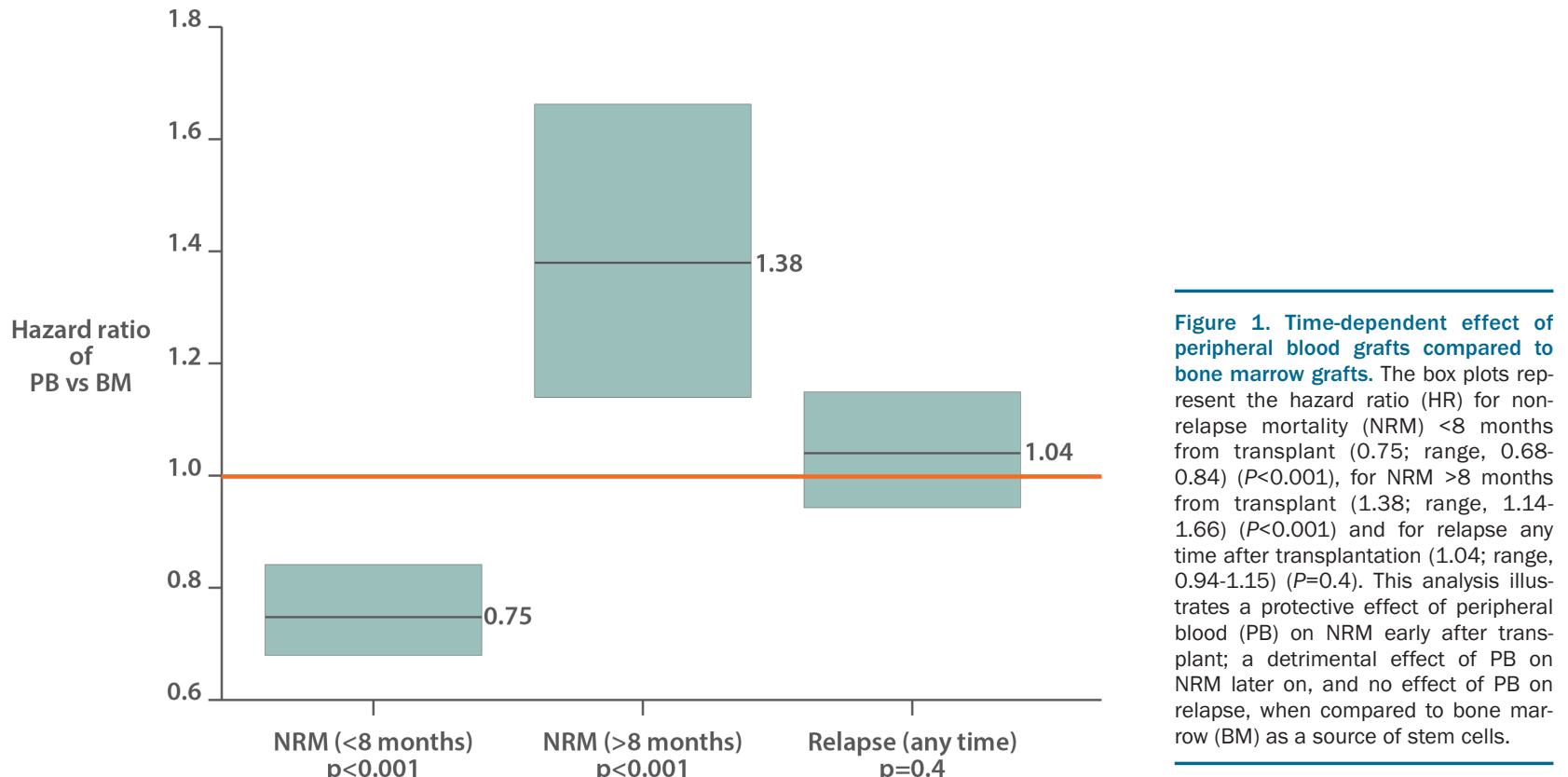

reduced deaths from other causes, and non-relapse mortality is significantly increased in the long-term.

Another debated issue is the comparison between reduced intensity and myeloablative conditioning regimens, and their effect on relapse and survival.4-5 The authors found that reduced intensity conditioning regimens protect patients from early non-relapse mortality (as expected), but this effect is lost after 4 months, and its competing event, relapse, unfortunately, increases constantly over time. Thus, when using a reduced intensity conditioning regimen, the clinician must be aware that the beneficial effect is short-lived and that in the longterm there is no protection against non-relapse mortality, with significantly greater risk of relapse.

In the era of personalized medicine the statistical approach suggested by Fuerst et al. provides a tool to disentangle the effects of different transplant components. This in turn gives new answers, sometimes unexpected, to important questions, such as the lack of reduced relapse risk using peripheral blood cells, or the significantly increased risk of relapse with reduced intensity condi- tioning regimens. A better understanding of these components lays the basis for a reconsideration of transplant protocols and the design of tailored clinical trials.

\section{References}

1. Fuerst D, Frank S, Mueller C, et al. Competing-risk outcomes after hematopoietic stem cell transplantation from the perspective of timedependent effects. Haematologica. 2018;103(9):1527-1534.

2. Friedrichs B, Tichelli A, Bacigalupo A, et al. Long-term outcome and late effects in patients transplanted with mobilised blood or bone marrow: a randomised trial. Lancet Oncol. 2010;11(4):331-338.

3. Anasetti C, Logan BR, Lee SJ, et al. Peripheral-blood stem cells versus bone marrow from unrelated donors. N Engl J Med. 2012;367(16):14871496.

4. Bornhäuser M, Kienast J, Trenschel R, et al. Reduced-intensity conditioning versus standard conditioning before allogeneic haemopoietic cell transplantation in patients with acute myeloid leukaemia in first complete remission: a prospective, open-label randomised phase 3 trial. Lancet Oncol. 2012;13(10):1035-1044.

5. Scott BL, Pasquini MC, Logan BR, et al. Myeloablative versus reducedintensity hematopoietic cell transplantation for acute myeloid leukemia and myelodysplastic syndromes. J Clin Oncol. 2017;35 (11):1154-1161. 